\title{
Cuantificación de biomasa y valor energético de renovales de Quercus ilex en condiciones mediterráneas
}

\author{
Quantification of biomass and energetic value of young natural regenerated stands \\ of Quercus ilex under Mediterranean conditions
}

\author{
Harald Fernández-Puratich ${ }^{\text {a*}}$, José Vicente Oliver-Villanueva ${ }^{b}$ \\ *Autor de correspondencia: a Universidad de Talca, Facultad de Ingeniería, Centro de Sistemas de Ingeniería Kipus, \\ Camino a Los Niches s/n, Curicó, Chile, tel.: (56) 75220 1793, hfernandezp@utalca.cl

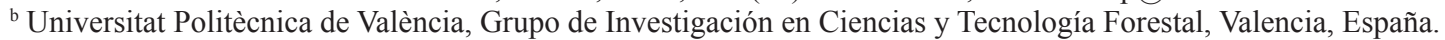

\begin{abstract}
SUMMARY
Forest biomass is key to Spain's energy policy. National, regional and local strategies, development programs of management plans and biomass and quantification of the most important species are fundamental. The objective of this research was to quantify and to analyze the calorific value of biomass of holm oak (Quercus ilex subsp. ballota) under Mediterranean conditions. To do this, several natural regenerated plots were selected in the Valencian Community (Eastern Spain). The main obtained results show that the average volume and weight per tree are $0.045 \mathrm{~m}^{3}$ tree ${ }^{-1}$ and $0.033 \mathrm{Mg}$ tree $^{-1}$ respectively. In addition, the form factor was determined (0.66). With an average stand density of 3,200 trees ha ${ }^{-1}$, the total potential biomass is $105.4 \mathrm{Mg} \mathrm{ha}^{-1}$. With the results obtained, a function of stem biomass related to the variables DBH (diameter at breast height) and Vf (volume of stem) has been adjusted with significantly high determination level and low estimation errors. This function can be used for the prediction of biomass quantification in natural regenerated holm oak pure stands in the Mediterranean area. Laboratory results show an energetic value of $17.0 \mathrm{MJ}^{\mathrm{k}} \mathrm{kg}^{-1}$ biomass. Therefore, the total potential energy is $513 \mathrm{MJ}_{\text {tree }}^{-1}$ and $1.8 \mathrm{GJ} \mathrm{ha}^{-1}$. Considering the large forest areas and the potential resources derived from clearings, thinning and fire prevention silvicultural treatments, the holm oak has the aptitude to be considered a valid raw material for energy use (mainly thermal) in Mediterranean rural areas.
\end{abstract}

Key words: Quercus ilex, biomass, quantification, energetic value.

\section{RESUMEN}

La biomasa forestal es clave en la política energética de España. Siendo fundamentales sus estrategias nacionales, regionales y locales, los programas de desarrollo de planes de manejo y la cuantificación de biomasa de las especies más importantes. Así, el objetivo de la investigación fue cuantificar y analizar el valor energético de la biomasa de encina (Quercus ilex subsp. ballota) en condiciones Mediterráneas. Para ello, varias parcelas de regeneración natural fueron seleccionadas en la Comunidad Valenciana (Este de España). Los principales resultados mostraron un volumen y peso promedio por árbol de $0,045 \mathrm{~m}^{3}$ árbol-1 y $0,033 \mathrm{Mg}^{-1}$ ábol ${ }^{-1}$ respectivamente. Además, se determinó el factor de forma $(0,66)$. Con una densidad de masa promedio de 3.200 árboles ha ${ }^{-1}$ la biomasa potencial es de $105,4 \mathrm{Mg} \mathrm{ha}^{-1}$. Con los resultados, se ajustó una función de biomasa fustal relacionada con el diámetro a la altura del pecho y volumen del fuste con alto coeficiente de determinación y bajos errores de estimación. Esta función es predictiva para la cuantificación de biomasa en bosques puros de regeneración natural de encinas del área Mediterránea. En laboratorio se obtuvo un valor energético de biomasa

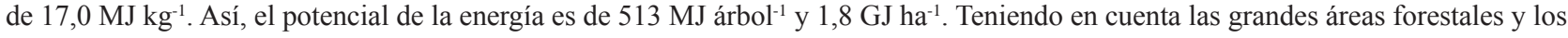
recursos potenciales derivados de desmontes, clareos y tratamientos silvícolas de prevención de incendios, la encina tiene la aptitud para considerarse como materia prima válida para su uso energético (principalmente térmico) en las áreas rurales mediterráneas.

Palabras clave: Quercus ilex, biomasa, cuantificación, valor energético.

\section{INTRODUCCIÓN}

La gestión sostenible y la extracción de la biomasa forestal para usos energéticos en condiciones mediterráneas están fuertemente condicionadas por los costos de cosecha, así como la logística de suministro para los diferentes sectores que la consumen (Berndes 2003, Gómez 2008, IMIDA 2012). Las operaciones forestales, el tipo y la clasificación de materia prima, astillado in situ y transporte desde el bosque a las plantas se ven muy influenciados por parámetros forestales de infraestructura claves en condiciones mediterráneas: estructura de la masa forestal (especies disponibles), en forma pura o mezclada y regular o irregular, pendiente, conservación del suelo y el riesgo de erosión, la accesibilidad vial, la conservación de áreas protegidas, etc. (IDAE 2007, Novo 2008). Estas limitaciones económicas y ambientales exigen que los actuales proyectos forestales industriales, que integran la gestión 
sostenible y el aprovechamiento energético de la biomasa forestal, estén confinados a áreas limitadas en el ámbito local o subregional (Enersilva 2007).

Los bosques mediterráneos tienen un gran potencial de biomasa leñosa rentable que pueden aportar con aumentar la oferta de especies forestales de donde se puede extraer diversas maderas duras y blandas que han sido para calefacción durante décadas en las zonas rurales de España (Frías 1985, IDAE 2007). La biomasa de base forestal es un elemento clave de la política energética actual en España en cuanto a las fuentes de energía renovables (PANER 2010). En consecuencia, son fundamentales en el desarrollo de este sector las estrategias forestales actuales a nivel nacional y regional y los programas operativos derivados de la necesidad de incluir el desarrollo de adecuados planes de manejo sustentables y la cuantificación de biomasa real de las especies forestales más importantes (PATFOR 2011).

En esta investigación, Quercus ilex L. (encina) ha sido seleccionada debido a que es la especie de madera dura con más existencias en la Península Ibérica. Sólo en España, los encinares ocupan 1.423.000 ha, excluyendo dehesas (Rivas-Martínez y Penas 2003, Rodà et al. 2009). Esto representa el $10 \%$ del área total de bosques en España (IFN3 2008). Los bosques de encinas principalmente son rodales regenerados naturalmente (Pulido et al. 2001). Extensos ecosistemas agro-forestales de encinas, generalmente en forma de dehesas, en las regiones del interior de España tienen un territorio adicional de 1.548.991 ha (Díaz y Pulido 2009). En la Comunidad Valenciana, la encina (21 \% de la superficie forestal) es, junto con Pinus halepensis Mill. $(57 \%)$, la base de la estructura forestal de la región (Generalitat Valenciana 1999, IFN-3 2007, PATFOR 2011).

Mientras Pinus halepensis, Pinus pinaster Ait. y Pinus sylvestris L. han sido estudiados en términos de cuantificación de biomasa y análisis de valor energético (Fisher et al. 1986, Skilling D. 1990, Nuñez-Regueira et al. 1996, Elfving y Kiviste 1997, Márquez-Montesino et al. 2001, Bravo-Oviedo et al. 2004, Barrio-Anta et al. 2006, Godoy et al. 2007, Dimitrakopoulos et al. 2011, Mukhortova 2012, Mouissa y Fournier 2013), la encina ha sido poco estudiada en este contexto como masa boscosa pura. Por lo tanto, el objetivo general de esta investigación es el estudio del potencial de la biomasa de encina contenida en masas puras regeneradas naturalmente en condiciones mediterráneas para su uso como biocombustible sólido, estableciendo si es posible la predicción de la producción de biomasa y potencial energético de la masa boscosa bajo estudio para una futura orientación energética del plan de manejo de bosques jóvenes de regeneración natural de encina. Para lograr este objetivo, la investigación tiene dos objetivos específicos: i) desarrollar una ecuación predictiva para la cuantificación de biomasa de encina en masas puras regenerada naturalmente en función de los parámetros morfológicos simples (análisis fustal dendrométrico y determinación de los coeficientes morfológicos) y ii) analizar el poder calorífico del material leñoso obtenido con el fin de evaluar el potencial energético de este recurso forestal.

\section{MÉTODOS}

Área de estudio. El trabajo se desarrolló en la región Este de España, en la Comunidad Valenciana, Provincia de Castellón, Comarca de Els Ports (Morella). La presencia de encina en bosques puros y mixtos en esta Comunidad Autónoma se muestra en la figura 1 . Más del $70 \%$ de las poblaciones puras se encuentran en la parte norte de la región, en la provincia de Castellón, en el nivel superior, con un gradiente altitudinal entre 800 a $1.200 \mathrm{~m}$ s.n.m.

Una parte importante de esta masa forestal es una regeneración natural pura de encina, después de grandes y devastadores incendios forestales hace aproximadamente 30 años. Cabe señalar que todas las encinas seleccionadas fueron identificadas en la unidad de Silvicultura de la Universidad Politécnica de Valencia como Quercus ilex L. subsp. ballota (Desf.) Samp. Es por ello por lo que se incluye la subespecie en el texto.

Selección de parcelas de muestreo. Después de la identificación de las zonas forestales más representativas principalmente a través del Tercer Inventario Nacional Forestal (IFN3 2008), el muestreo se llevó a cabo en una altitud media de $1.100 \mathrm{~m}$ s.n.m. en una masa boscosa coetánea de aproximadamente 30 años de edad. Los siguientes parámetros fueron documentados en terreno para cada masa forestal (cuadros $1 \mathrm{y}$ 2): densidad (árboles ha ${ }^{-1}$ ), altitud media (m), pendiente (\%), exposición, DAP promedio $(\mathrm{cm})$ y distribución de clases de DAP (\%). Valores medios y desviación estándar se calcularon para cada rodal, seleccionando cinco parcelas representativas de un tamaño mínimo de parcela de 0,25 ha. A continuación el cuadro 1 describe en detalle cada parcela evaluada.

Selección de árboles muestreados. Un completo inventario terrestre se llevó a cabo para cada parcela de muestreo documentando la densidad de población, DAP y altura. Para la selección de los árboles muestreados, se consideró, principalmente, que un gran número de árboles por parcela (tamaño de muestra) permiten una alta significación de los grupos homogéneos en la muestra (Argibay 2009). Sobre la base de que, con el fin de optimizar la representatividad estadística, por un lado, y la economía de la investigación, por el otro, se aplicó el método de Hapla y Saborowski (1984) para la determinación del tamaño de la muestra:

$\mathrm{N} \min >\frac{\left(\mathrm{z}^{2} \times \mathrm{s}^{2}\right)}{\mathrm{I}^{2}}$

Donde,

$\mathrm{N}$ min = Mínimo de la muestra (árboles por parcela)

$\mathrm{z}=1,96$ es el valor crítico de la distribución normal estándar para el nivel de significación 2,5 \% (Sachs 1984).

$\mathrm{s}=$ desviación estándar

$1=$ precisión absoluta deseada, que se define como

$1=0,01 * d * x \min$

$\mathrm{d}=$ dadas precisión relativa de $95 \%$, de forma que $\mathrm{d}=5$

xmin $=$ valor promedio más bajo de todas las muestras . 


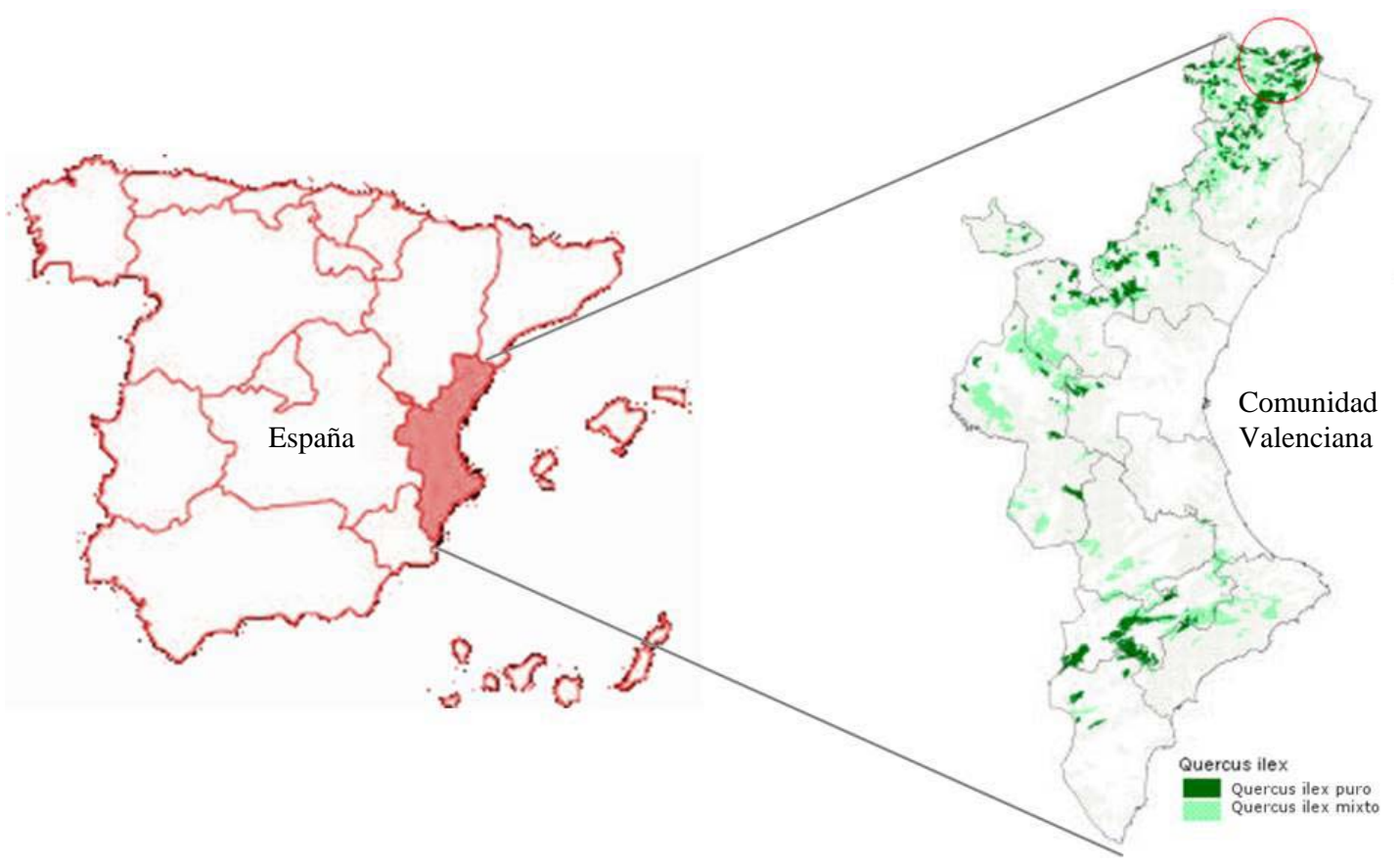

Figura 1. Mapa de ubicación de la Comunidad Valenciana en España y distribución de Quercus ilex en bosques puros y mixtos en la Comunidad Valenciana y localización de los puntos de muestreo seleccionados (PGOF 2004).

Location map of the Comunidad Valenciana in Spain and distribution of Quercus ilex on pure and mixed forests in the Valencian Community (Spain) and localization of the selected sampling plots (PGOF 2004).

Cuadro 1. Descripción de parcelas muestreadas. Description of plots sampled.

\begin{tabular}{cccccc}
\hline Parcela & Superficie (ha) & Densidad (árboles ha $\mathrm{a}^{-1}$ ) & Altitud (m snm) & Pendiente (\%) & Exposición \\
\hline 1 & 0,31 & 2.990 & 1.078 & 24 & NO \\
2 & 0,34 & 3.250 & 1.096 & 30 & NO \\
3 & 0,25 & 3.010 & 1.109 & 34 & NO \\
4 & 0,37 & 3.450 & 1.107 & 31 & $\mathrm{~N}$ \\
5 & 0,33 & 3.300 & 1.117 & 36 & $\mathrm{~N}$ \\
\hline
\end{tabular}

Los resultados de los análisis preliminares definieron un mínimo muestral de 15 árboles por parcela, obteniéndose un mínimo total de 75 árboles muestreados (figura 2).

Para la selección de los árboles individuales, se realizó un análisis de la distribución normal de DAP y altura, obteniendo valores medios y la desviación estándar. Todos los árboles individuales que estaban dentro del rango de confianza único (media \pm desviación estándar) tanto en DAP como en altura, han sido considerados como representativos y fueron marcados. De este muestreo, 15 árboles se seleccionaron por sistema aleatorio simple, evitando influencias de sus bordes, árboles malformados o enfermos.

Análisis morfológico del fuste. El DAP representativo se obtuvo siguiendo la metodología de Siostrzonek. Una vez determinado el diámetro máximo con una forcípula, el diámetro representativo se determinó por la rotación de $22,5^{\circ}$ en sentido horario. La altura se midió con hipsómetro.

El volumen de cada fuste individual (Vi) se determinó a partir de un factor de forma $\mathrm{f}$ (ecuación 2), que es la relación entre el volumen del fuste real y el modelo de volumen de un cilindro con el diámetro de referencia, en este caso el DAP (Prodan 1997).

$$
f=\frac{\text { Volumen real de la estructura }}{\text { Volumen del modelo }}
$$

El volumen real de la estructura (fuste) fue determinado, a través de la fórmula de Hohenadl, en cinco secciones de un metro de largo por árbol: 

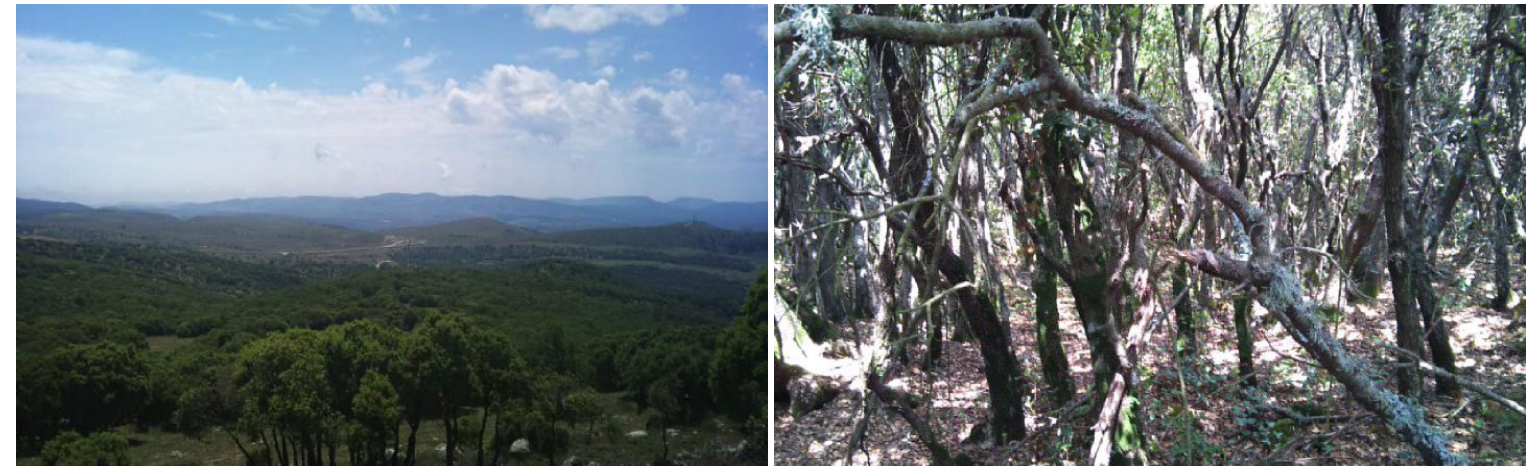

Figura 2. Selección de árboles individuales representativos de las parcelas de muestreo sin manejo. Selection of representative individual trees in the sampling plots of unmanaged.

$\mathrm{V}=\frac{\Pi}{4} \times \mathrm{L} \times\left(\mathrm{d}_{0,1}^{2}+\mathrm{d}_{0,3}^{2}+\mathrm{d}_{0,5}^{2}+\mathrm{d}_{0,7}^{2}+\mathrm{d}_{0,9}^{2}\right)$

Donde,

$\mathrm{V}=$ volumen del fuste en $\mathrm{m}^{3}$,

$\mathrm{L}=$ largo de la sección en $\mathrm{m}$,

$\mathrm{d}=$ diámetro medio de la sección en $\mathrm{cm}$.

El factor de forma es un parámetro característico para la especie y de la clase a que se refiere el diámetro del fuste. Sin embargo, por razones estadísticas, la media y la dispersión se determinó para cada parcela.

Análisis de la relación entre diámetro de fuste y volumen. El volumen fustal se obtuvo de cada árbol a través del DAP y la altura como parámetros de referencia tal como indica el cuadro 2 para cada parcela. Una vez obtenidos estos resultados se han llevado a cabo una relación lineal y exponencial y un análisis de regresión entre volumen y DAP. Lo que se busca es predecir el volumen del fuste $(V)$, por medio de una ecuación de regresión altamente significativa, incluyendo sólo la variable DAP y un factor de forma constante entre las parcelas evaluadas.

Análisis de la energía potencial. La densidad básica que es el cociente entre la masa en estado anhidro y el volumen de la madera en estado verde. Por ser la masa numéricamente igual al peso, con fines prácticos se determinó el peso específico aparente básico que es el cociente entre peso anhidro y volumen verde en lugar de la densidad básica, así se ha determinado en laboratorio de acuerdo con la Norma Europea EN 56531, siendo el resultado utilizado como densidad básica. Las muestras de cada árbol individual seleccionado corresponden a tarugos de 10 a $15 \mathrm{~cm}$ que han sido extraídos a la altura del pecho y transportados al laboratorio para el análisis. Cabe destacar que el valor de densidad básica corresponde a biomasa que contiene madera y corteza, esta última no mayor a un $5 \%$ del total del material analizado.
Cuadro 2. Descripción de parámetros dendrométricos en parcelas muestreadas.

Description of dendrometric parameters of sampled plots.

\begin{tabular}{ccc}
\hline \multirow{2}{*}{ Parcela } & \multicolumn{2}{c}{ Parámetros } \\
\cline { 2 - 3 } & DAP $(\mathrm{cm})$ & Altura $(\mathrm{m})$ \\
\hline 1 & 13,5 & 6,0 \\
2 & 12,2 & 5,8 \\
3 & 12,1 & 5,9 \\
4 & 10,7 & 5,7 \\
5 & 11,2 & 5,5 \\
\hline
\end{tabular}

A partir de la fórmula de volumen en metros cúbicos y conociendo la densidad de la madera se confeccionó una ecuación para calcular la biomasa contenida por árbol y luego por superficie al conocer la densidad de árboles por hectárea.

Se realizó la prueba t de Student para analizar si existen diferencias de los valores calculados de biomasa contenida por árbol y por hectárea entre dos subclases diametrales $($ DAP $<10 \mathrm{~cm} \mathrm{y}>10 \mathrm{~cm})$.

El poder calorífico a $0 \%$ de contenido de humedad de la madera ha sido también determinado en el laboratorio para todos los árboles seleccionados con muestras extraídas a la altura del pecho. Este análisis se ha llevado a cabo siguiendo la Norma Europea EN 14918. Siendo el valor medio de poder calorífico inferior (PCI) de $17,0 \mathrm{MJ} \mathrm{kg}^{-1}$ o 17,0 GJ $\mathrm{Mg}^{-1}$ (biomasa seca). Este valor se determinó en el laboratorio con el material obtenido de los árboles muestreados y fue incluido en la ecuación para calcular el PCI por árbol y luego por superficie al conocer la densidad de árboles por hectárea.

De esta manera, el peso de la biomasa $(\mathrm{kg}$ o $\mathrm{Mg})$ y el poder calorífico total (MJ o GJ) se pueden calcular tanto para el árbol individual, así como para el rodal por hectárea siguiendo los modelos matemáticos determinados por los valores obtenidos para el volumen por árbol y por hectárea, obteniendo así nuevos modelos predictivos para la obtención de biomasa y potencial energético en esta especie. 


\section{RESULTADOS}

Diámetro y altura. El DAP promedio de los 75 árboles representativos seleccionados fue de $11,9 \mathrm{~cm}$, con un coeficiente de variación de $26,1 \%$ (cuadro 3). La altura mostró un promedio de $5,8 \mathrm{~m}$ la cual varía muy poco con un coeficiente de variación del 7,9 \% (cuadro 3). La distribución del DAP de los árboles analizados mostró que el 70\% de los fustes estaban entre 7,0 cm y $14,0 \mathrm{~cm}$ (figura 3 ).

Factor de forma fustal. El factor de forma fustal promedio fue de 0,66 con un coeficiente de variación bajo, del orden del $10,7 \%$ (cuadro 4 ).

$V=0,005 * \mathrm{DAP}^{2}-0,0048 * \mathrm{DAP}+0,0226$

Volumen fustal. El volumen promedio del fuste sin ramas es $0,045 \mathrm{~m}^{3}$ árbol-1 (cuadro 5) y con una densidad de población de 3.200 árboles ha $^{-1}$ el volumen total promedio es de 144,8 $\mathrm{m}^{3} \mathrm{ha}^{-1}$. El volumen fustal total de las muestras tuvo una alta variación $(\mathrm{CV}=57,0 \%)$. Sin embargo, al subdividir la muestra en dos subclases diametrales (DAP $<10 \mathrm{~cm}$ y DAP $>10$ $\mathrm{cm}$ ), esta variación se redujo considerablemente (cuadro 5).

Cuadro 3. Estadísticas descriptivas de la distribución de las clases de diámetro del fuste $(\mathrm{cm})$ y altura $(\mathrm{m})$ de 75 árboles.

Descriptive statistics of the distribution of stem diameter classes $(\mathrm{cm})$ and tree height $(\mathrm{m})$ of 75 trees.

\begin{tabular}{crc}
\hline \multirow{2}{*}{ Parámetro } & \multicolumn{2}{c}{ Variable } \\
\cline { 2 - 3 } & DAP & Altura \\
\hline Mínimo & 7,5 & 4,5 \\
$-\sigma d$ & 8,8 & 5,3 \\
Promedio & 11,9 & 5,8 \\
$+\sigma d$ & 15,0 & 6,2 \\
Máximo & 17,4 & 7,2 \\
CV (\%) & 26,1 & 7,9 \\
\hline
\end{tabular}

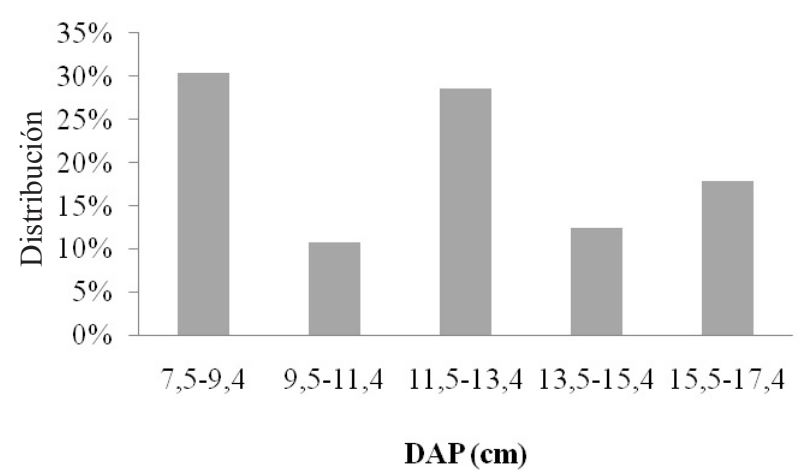

Figura 3. Distribución (\%) del DAP en los árboles analizados de Quercus ilex.

Distribution (\%) of DBH in the analysed Quercus ilex trees.
Relación entre el DAP y volumen fustal. La ecuación 4 que fue la más significativa obtenida después del análisis de regresión múltiple (figura 4).

Se obtuvo la siguiente ecuación para calcular la biomasa fustal por árbol, teniendo en cuenta una densidad básica de la madera de $0,732 \mathrm{Mg} \mathrm{m}^{-3}$.

$\mathrm{V}=0,005 * \mathrm{DAP}^{2}-0,0048 * \mathrm{DAP}+0,0226$

Teniendo en cuenta una densidad de población media de 3.200 árboles ha ${ }^{-1}$, la siguiente ecuación permite calcular la cantidad total de biomasa por hectárea.

Biomasa $\left(\mathrm{kg} \mathrm{árbol}^{-1}\right)=0,366 * \mathrm{DAP}^{2}-3,5136 * \mathrm{DAP}+16,5432$

Debido a las variaciones observadas en el volumen del fuste de las dos subclases diametrales (DAP $<10 \mathrm{~cm}$ y $>$ $10 \mathrm{~cm}$ ), las ecuaciones 5 y 6 se ajustaron para estas dos subclases (cuadro 6).

Incluyendo todos los árboles de la muestra con un DAP promedio de $11,9 \mathrm{~cm}$ y un volumen de $0,045 \mathrm{~m}^{3}$ árbol $^{-1}$, la biomasa promedio calculada fue de $30,2 \mathrm{~kg}$ árbol $^{-1}$ y 96,5 $\mathrm{Mg} \mathrm{ha}^{-1}$.

Cuadro 4. Estadísticas descriptivas del factor de forma fustal $\left(f_{\mathrm{v}}\right)$. Descriptive statistics of the stem form factor $\left(\boldsymbol{f}_{\mathrm{v}}\right)$.

\begin{tabular}{cc}
\hline Parámetro & Valor \\
\hline $\mathrm{N}$ & 75 \\
Mínimo & 0,55 \\
$-\sigma d$ & 0,59 \\
Promedio & 0,66 \\
$+\sigma d$ & 0,73 \\
Máximo & 0,80 \\
CV $(\%)$ & 10,7 \\
\hline
\end{tabular}

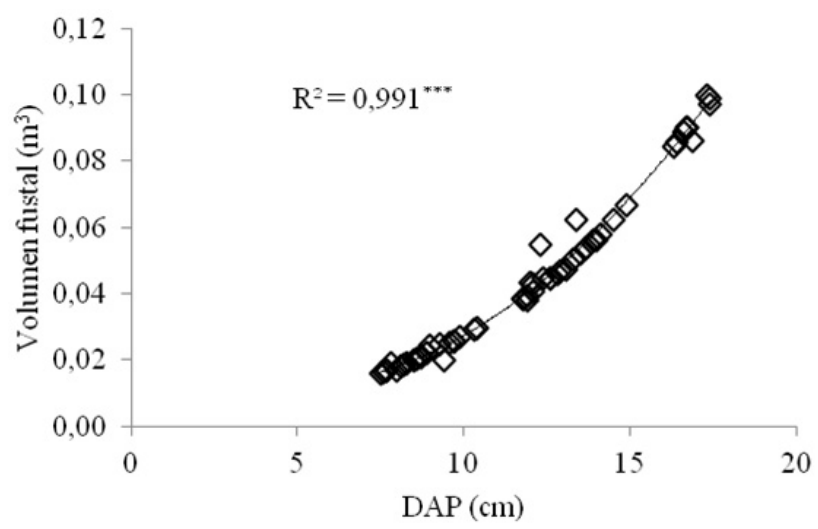

Figura 4. Relación entre el DAP y volumen fustal. Relationship between DBH and stem volume. 
Cuadro 5. Volumen fustal $\left(\mathrm{m}^{3}\right.$ árbol $\left.^{-1}\right)$. Stem volume $\left(\mathrm{m}^{3}\right.$ tree $\left.{ }^{-1}\right)$.

\begin{tabular}{cccccccc}
\hline DAP & N & Mínimo & - of & Promedio & + of & Máximo & CV (\%) \\
\hline$<10,0 \mathrm{~cm}$ & 30 & 0,016 & 0,017 & 0,021 & 0,024 & 0,027 & 17,1 \\
$>10,0 \mathrm{~cm}$ & 45 & 0,029 & 0,038 & 0,060 & 0,082 & 0,100 & 36,4 \\
Total & 75 & 0,019 & 0,029 & 0,045 & 0,071 & 0,086 & 57,0 \\
\hline
\end{tabular}

Cuadro 6. Resumen de la biomasa contenida ( $\mathrm{kg}$ y $\mathrm{Mg}$ ) en los bosques naturales regenerados de Quercus ilex a una densidad de población media de 3.200 árboles ha ${ }^{-1}$.

Summary of the biomass contained $(\mathrm{kg}$ and $\mathrm{Mg}$ ) in natural regenerated forests of Quercus ilex with an average stocking density of 3.200 trees $\mathrm{ha}^{-1}$.

\begin{tabular}{|c|c|c|c|}
\hline $\begin{array}{l}\text { DAP } \\
(\mathrm{cm})\end{array}$ & $\begin{array}{l}\text { Volumen } \\
\left(\mathrm{m}^{3} \text { árbol }^{-1}\right)\end{array}$ & $\begin{array}{c}\text { Biomasa } \\
\left(\mathrm{kg} \mathrm{árbol}^{-1}\right)\end{array}$ & $\begin{array}{l}\text { Total biomasa } \\
\left(\mathrm{Mg} \mathrm{ha}^{-1}\right)\end{array}$ \\
\hline$<10,0$ & 0,021 & 15,2 & 48,6 \\
\hline$\geq 10,0$ & 0,060 & 43,9 & 140,4 \\
\hline Promedio & 0,045 & 30,2 & 96,5 \\
\hline
\end{tabular}

Según la prueba $t$ de Student para biomasa contenida por árbol y por hectárea entre las dos subclases diametrales (DAP $<10 \mathrm{~cm} \mathrm{y}>10 \mathrm{~cm}$ ) existen diferencias significativas entre sus medias para un nivel de confianza del 95,0 $\%$. Estos resultados pueden servir de base para un plan de manejo, por ejemplo, para limpieza y clareo selectivo de los árboles de menor DAP.

Una vez determinado el contenido de biomasa es posible obtener la cantidad de energía por árbol o por hectárea (PCI de 17,0 MJ kg-1). Para ello, se determinó la siguiente ecuación para calcular el PCI por árbol:

$\mathrm{PCI}\left(\mathrm{GJ}\right.$ árbol $\left.^{-1}\right)=0,00622 * \mathrm{DAP}^{2} \cdot 0,05973 * \mathrm{DAP}+0,2812$

Para la predicción del PCI por hectárea y a una densidad de población media de 3.200 árboles ha ${ }^{-1}$, se ha definido la siguiente ecuación:

\section{$\operatorname{PCI}\left(\mathrm{GI} \mathrm{ha} \mathrm{a}^{-1}\right)=19,91 * \mathrm{DAP}^{2} \cdot 191,14^{*} \mathrm{DAP}+899,95$}

El cuadro 7 muestra el cálculo del potencial energético de las dos clases diamétricas (DAP $<10 \mathrm{~cm} \mathrm{y}>10 \mathrm{~cm}$ ). El potencial energético medio obtenido en esta investigación fue de $0,513 \mathrm{GJ}$ ábol $^{-1}$ y 1.640,3 $\mathrm{GJ} \mathrm{ha}^{-1}$, que corresponde a biomasa fustal en base seca.

\section{DISCUSIÓN}

A diferencia de otras especies de árboles y áreas geográficas, los árboles de encina estudiados, corresponden a una masa forestal pura sin mucha intervención antrópica en las últimas décadas, ubicada en el mismo distrito en condiciones similares de sitio. Sin embargo, la condición del bosque puro de estos rodales con regeneración natural comparte características similares de homogeneidad con las repoblaciones forestales mono-específicos, ya que tiene una masa uniforme de una sola especie, coetáneos y en las mismas condiciones de sitio que se traduce en una baja variabilidad del DAP, lo que es de esperar en masas boscosas regeneradas naturalmente después de grandes incendios forestales en condiciones del sitio similares. Las superficies de bosques de encinas están aumentando considerablemente en la zona mediterránea de la península ibérica debido a la intensiva regeneración natural después de los incendios forestales, así como en las antiguas tierras agrícolas. Hoy en día, estos recursos no se utilizan para fines materiales.

Para evaluar el estado actual de los renovales de encina y adoptar las decisiones adecuadas de gestión es necesario la obtención de una descripción cuantitativa de estos bosques, que son posibles de obtener a partir de las distribuciones de frecuencia y diámetros, debido a la alta proporcionalidad entre esta variable y la información obtenida a partir de un inventario forestal (Prodan et al. 1997). Por lo tanto, la importancia de determinar las clases diamétricas es que el DAP es una medición directa de las dimensiones de los árboles y constituye un input básico para el cálculo del área basal, volumen del fuste, biomasa de los árboles, cobertura, etc. (FAO 1997).

En varios estudios se proponen diferentes diámetros de referencia para la utilización como recurso energético de la biomasa de las diferentes especies forestales mediterráneas, principalmente de coníferas. Merino et al. (2005) proponen un diámetro mínimo de $7,0 \mathrm{~cm}$ y Brañas et al. (2000) toma como diámetros de referencia de $4,0 \mathrm{~cm}$ para el mismo propósito. Coincidiendo con el primer autor en los diámetros mínimos que contempla este estudio. Estrategias actuales y planes forestales en las regiones mediterráneas han ampliado este límite. Por ejemplo, PATFOR (2011) observó que todos esos árboles de diámetro con corteza bajo 23,0 cm son susceptibles de ser considerados integralmente como recurso bioenergético. Es decir, el conjunto de su biomasa aérea ahora se considera como la biomasa forestal residual. En este estudio, un diámetro mínimo de $7,0 \mathrm{~cm}$ se ha considerado para el potencial industrial o el uso de energía que coincide con Merino et al. (2005). 
Cuadro 7. Resumen de potencial energético por árbol y por superficie en rodales puros de Quercus ilex. Summary of the energy potential per tree and per surface in pure stands of Quercus ilex.

\begin{tabular}{|c|c|c|c|c|}
\hline $\mathrm{DAP}(\mathrm{cm})$ & $\begin{array}{l}\text { Biomasa } \\
\left(\mathrm{kg} \mathrm{árbol}^{-1}\right)\end{array}$ & $\begin{array}{l}\text { Total biomasa } \\
\left(\mathrm{Mg} \mathrm{ha}^{-1}\right)\end{array}$ & $\begin{array}{c}\text { PCI } \\
\left(\mathrm{GJ}_{\text {árbol }}^{-1}\right)\end{array}$ & $\begin{array}{c}\text { Potencial energético } \\
\qquad\left(\mathrm{GJ} \mathrm{ha}^{-1}\right)\end{array}$ \\
\hline$<10,0$ & 15,2 & 48,6 & 0,258 & 826,2 \\
\hline \multirow[t]{2}{*}{$\geq 10,0$} & 43,9 & 140,4 & 0,746 & $1.801,5$ \\
\hline & & Promedio & 0,513 & $1.640,3$ \\
\hline
\end{tabular}

Según Rodà et al. (2009), el 96,7 \% de encina en España están por debajo de $22,5 \mathrm{~cm}$ de DAP que es un valor máximo similar al encontrado en este estudio (cuadro 2). Siendo, el lento crecimiento radial de esta especie en condiciones mediterráneas con altitudes superiores a $1.000 \mathrm{~m}$ s.n.m. explicado por las limitaciones de sitio en las zonas marginales donde ha sido desplazada o aislada por la actividad agrícola. Pero la razón más importante es la alta densidad de población (3.200 árboles ha ${ }^{-1}$ ) resultado de la ausencia de tratamientos silviculturales (clareos o limpieza, raleos) o alteraciones mínimas, incluso desde su origen (Cruz et al. 2009). Además, las grandes dimensiones de fuste en el rodal se han eliminado debido a que históricamente se ha usado como leña (Ortego et al. 2010).

Con respecto al factor de forma fustal este se mantiene prácticamente constante en las parcelas evaluadas existiendo un coeficiente de variación entre parcelas de tan solo $10,7 \%$, teniendo un valor promedio de 0,66 cifra coincide que es muy similar a la obtenida por otros autores. Serrada-Hierro y San Miguel (2008) calcularon un factor de forma de 0,69 para distintas edades de encinas. IFN3 (2008) especifica un factor de forma de 0,74. Sin embargo, estas diferencias entre los valores de referencia y los valores obtenidos en esta investigación no se puede evaluar como significativo.

En general, estas pequeñas diferencias se pueden atribuir a tres causas:

a) Los árboles representativos analizados tienen las mismas características morfológicas. La selección de la muestra ha incluido grupos homogéneos de ambos DAP y altura.

b) Los árboles representativos analizados crecen en zonas con similares condiciones fisiográficas. Se puede aceptar que la forma del fuste varíe, lo que depende en gran medida de las condiciones ambientales del sitio (Donoso 1995).

c) La densidad de población (árboles por hectárea) en las parcelas de muestreo es muy alta en comparación con otros rodales de encinas manejados.

El volumen promedio del fuste sin ramas obtenido en este estudio (cuadro 5) coincide con García et al. (2009) que obtuvieron valores muy similares $\left(0,050 \mathrm{~m}^{3}\right.$ árbol $\left.{ }^{-1}\right)$ de encina en las masas en la región de Castilla-La Mancha (España). El volumen fustal total de las muestras tiene una alta variación. Sin embargo, si la muestra se subdivide en dos subclases diametrales (DAP $<10 \mathrm{~cm} \mathrm{y}>10 \mathrm{~cm}$ ), esta variación se reduce considerablemente. Los rodales jóvenes de regeneración natural de encina son homogéneos en la morfología del árbol (DAP y altura), pero de culminación rápida de crecimiento en altura en comparación con el crecimiento diametral afecta directamente la variabilidad del volumen de fuste como potencial de biomasa (González y Ibarz 1998). Siendo esta aproximación a la culminación en altura una situación que se está dando en las parcelas estudiadas, lo que es corroborado por SerradaHierro et al. (2004) quienes, en similares condiciones de sitio a este estudio, obtienen una altura promedio de 6,8 m (desviación estándar $\pm 0,9$ ) y DAP de $38,9 \mathrm{~cm}$ (desviación estándar de $\pm 15,0)$ para árboles que van entre 70 y 251 años de edad, en contraste, los árboles de este estudio tienen una altura de 5,8 m, pero un DAP de tan solo 11,9 $\mathrm{cm}$ (cuadro 3) para masas boscosas de aproximadamente 30 años de edad. Lo que da cuenta del escaso aumento en altura con respecto al importante aumento del DAP que se podría esperar en el futuro en las parcelas estudiadas.

La variación volumétrica es mayor en los árboles más delgados. Así, los árboles seleccionados con diámetros más pequeños muestran un coeficiente de variación inferior y los árboles con diámetros mayores muestran uno mayor.

La relación obtenida del modelo de predicción entre el DAP y volumen fustal (figura 4) es muy recomendable para su aplicación práctica. Este resultado no es inesperado, debido a la homogeneidad de las parcelas de muestreo y el análisis de los anteriores árboles representativos seleccionados. En consecuencia, este modelo de predicción se puede aplicar para rodales de encinas jóvenes puros de regeneración natural coetáneos (aproximadamente 30 años) y no manejados.

En esta investigación sólo se ajustaron ecuaciones para el potencial energético de la biomasa leñosa del fuste, sin considerar la biomasa leñosa contenida en las ramas, ya que los bosques de encinas en poblaciones puras de la cuenca Mediterránea son generalmente regeneraciones naturales después de incendios o tala rasa (Espelta 1996) que debido al aumento de la competencia en las densidades más elevadas, los árboles se ven obligados a buscar la luz, creciendo rápidamente en altura y el desarrollo de la poda natural (Perry 1985), que es lo que ocurre en esta masa boscosa, teniendo un bajo contenido de ramas por árbol. Sin embargo, cuando la densidad de población es reducida debido a 
los tratamientos silviculturales (raleos y claros), la competencia por la luz es menor y los árboles crecen lentamente en altura y con muchas ramas.

Además del desembosque y posterior transporte de las trozas que resulta ser más eficiente sin ramas (con un desrrame en bosque), el Institute Pinchot (2010) señala que las operaciones de limpieza y raleo en estos rodales normalmente dejan el material de la copa en el bosque para evitar una exportación importante de nutrientes. El material de la copa es normalmente astillado in situ y distribuido por todo el rodal con el fin de prevenir contra los incendios forestales (PNW 2010).

Los valores totales de biomasa aérea promedio obtenidos (cuadro 6) están en concordancia con estudios anteriores. Así, para un DAP promedio de $10,0 \mathrm{~cm}$ en encina, Susmel et al. (1976) obtuvieron $44 \mathrm{~kg}^{2}$ ábol $^{-1}$, Férrres et al. (1980) $42 \mathrm{~kg}$ árbol$^{-1}$, Canadell et al. (1988) $36 \mathrm{~kg}$ árbol ${ }^{-1}$. Por otra parte, Pérez y Esteban (2004) obtuvieron 31,4 kg árbol $^{-1}$ con un DAP de $11,3 \mathrm{~cm}$.

El resto de los componentes ramas y hojas (biomasa de copa) se deja en el monte, siendo utilizado solo el fuste para fines energéticos o materiales.

Una vez determinado el contenido de biomasa es posible obtener la cantidad de MJ o GJ por árbol o por hectárea. Para ello, las ecuaciones resultantes para la predicción del LHV por árbol y por hectárea resultan ser convenientes para su aplicación en pequeños rodales puros de encina con regeneración natural y en condiciones del sitio similares a las encontradas en este estudio.

Como referencia general, para establecer una comparación, Torres-Álvarez y Peña Cortés (2011) encontraron $3.488 \mathrm{GJ} \mathrm{ha}^{-1}$ para el tipo de bosque de frondosas coigüe-raulí-tepa (Nothofagus dombeyi (Mirb.) Oerst., Nothofagus alpina Poepp. et Endl., Kasser y Laureliopsis philippiana (Looser) Shodde) bajo condiciones de clima templado mediterráneo en Chile. Siendo este valor mayor al encontrado en este estudio (cuadro 7), lo que puede ser influido principalmente porque los autores han trabajado con el poder calorífico superior mientras que este estudio lo ha hecho con el poder calorífico inferior (PCI).

Sin embargo, el potencial energético por hectárea no es realmente utilizable por completo, porque el sistema de extracción de biomasa corresponde en general a clareos o raleos selectivos y no a tala rasa, así la cantidad de GJ ha-1 rentable depende del porcentaje de extracción de biomasa determinado con el plan de manejo forestal correspondiente. Para el previsto de raleos selectivos, los árboles con DAP menor pueden ser considerados primeramente.

\section{CONCLUSIONES}

El uso histórico como leña doméstica y la aptitud energética demostrada en este estudio abre una posibilidad de usarlo como fuente de energía alternativa.

El necesario raleo selectivo del cual se podría obtener la materia prima para destinarla a usos energéticos debería eliminar los árboles más pequeños con el fin de mejorar la estructura del bosque y el crecimiento diametral de los árboles en pie.

Basándose en el homogéneo factor de forma fustal observado y debido a la buena correlación obtenida entre DAP y volumen del fuste, la predicción de la producción de biomasa es posible. Teniendo en cuenta la densidad básica, el poder calorífico y la densidad de carga individual de los rodales, el resultado de los modelos de predicción ajustados se puede utilizar como base para una orientación energética del plan de manejo de bosques jóvenes de regeneración natural de encina. Esta fuente de biomasa puede complementar el suministro sostenido de otras biomasas de base forestal, principalmente de coníferas, para las plantas de bioenergía a nivel local o regional en el área mediterránea.

Además, en el futuro la idea de investigar, podría encaminarse hacia posibles diferencias entre Quercus ilex y Quercus rotundifolia y de sus subespecies (variablidad fiosionómica o genética) de sus características bioenergéticas, principalmente en el poder calorífico.

\section{AGRADECIMIENTOS}

Agradecemos las facilidades dadas por el Instituto de Ingeniería Energética de la Universitat Politècnica de València para la utilización de su laboratorio de biomasa en la determinación de la densidad y poder calorífico de la madera de encina.

\section{REFERENCIAS}

Acuña E. 2010. Estimating the bioenergy potential of Pinus radiata plantations in Chile. Ciencia e Investigación Agraria 37(1): 93-102.

Ayaril A, A Zubizarreta-Gerendiain, M Tome, J Tome, S Garchi, B Henchi. 2012. Stand, tree and crown variables affecting cone crop and seed yield of Aleppo pine forests in different bioclimatic regions of Tunisia. Forest Systems (1): 128-140.

Argibay J. 2009. Muestra en investigación cuantitativa. Subjetividad y Procesos Cognitivos 13: 13-29.

Barrio-Anta M, M A Balboa-Murias, F Castedo-Dorado, U Diéguez-Aranda, J G Álvarez-González. 2006. An ecoregional model for estimating volume, biomass and carbon pools in maritime pine stands in Galicia (north-western Spain). Forest Ecology and Management 223(1-3): 24-34.

Berndes G, M Hoogwijk, R van den Broek. 2003. The contribution of biomass in the future global energy supply: a review of 17 studies. Biomass Bioenergy 25: 1-28.

Brañas J, F González-Río, R Rodríguez-Soalleiro, A Merino. 2000. Biomasa maderable y no maderable en plantaciones de eucalipto, cuantificación y estimación. CIS-Madera 4: 72-75

Bravo-Oviedo A, M Del Río, G Montero. 2004. Site index curves and growth model for Mediterranean maritime pine (Pinus pinaster Ait.) in Spain. Forest Ecology and Management 201(2-3): 187-197.

Canadell J, M Riba, P Andres. 1988. Biomass Equations for 
Quercus ilex L. in the Montseny Massif, Northeastern Spain. Forestry 61(2): 137-147.

Cruz P, A Fernández, J Reque. 2009. Propuesta tipológica forestal para los bosques de Quercus pyrenaica de la comarca del Bierzo del noroeste de España, empleando análisis multivariante. Bosque 30(3): 180-191.

Díaz M, FJ Pulido. 2009. Dehesas perennifolias de Quercus spp. In Ministerio de Medio Ambiente, y Medio Rural y Marino eds. Bases ecológicas preliminares para la conservación de los tipos de hábitat de interés comunitario en España. Madrid, España. Ministerio de Medio Ambiente, y Medio Rural y Marino. 69 p.

Dimitrakopoulos AP, ID Mitsopoulos, A Kaliva. 2011. Short communication. Comparing flammability traits among firestricken (low elevation) and non fire-stricken (high elevation) conifer forest species of Europe: a test of the Mutch hypothesis. Forest Systems 22(1): 134-137

Donoso C. 1995. Ecología forestal: el bosque y su medio ambiente. Editorial Universitaria. 369 p.

Elfving B, A Kiviste. 1997. Construction of site index equations for Pinus sylvestris L. using permanent plot data in Sweden. Forest Ecology and Management 98: 125-134.

Enersilva. 2007. Promoción del uso de la biomasa forestal con fines energéticos en el suroeste de Europa (2004-2007). Opal SA. Consultado 23 jul. 2011. Disponible en http://enersilva. navegantes.info/articulos.php

Espelta JM. 1996. La regeneració de boscos d'alzina (Quercus ilex) i pi blanc (Pinus halepensis Milll.): estudi experimental de la resposta de les plántules a la intensitat de llum i la disponibilitat d'aigua. Tesis doctoral. Barcelona, España. Departament de Biologia Animal, de Biologia Vegetal i d'Ecologia. Universidad Autónoma de Barcelona. 255 p.

FAO (Food and Agriculture Organization, IT). 1997. Estimating Biomass and Biomass Change of Tropical Forests. 55 p. (Forestry Paper $\mathrm{N}^{\mathrm{o}}$ 134).

Ferres LL, F Roda, A Verdu, J Terradas. 1980. Estructura y funcionalismo de un encinar montano en el Montseny. Mediterránea 7: 139-166.

Frías J. 1985. Posibilidades de aprovechamiento económico de la biomasa residual. Agricultura y Sociedad 34: 219-236.

García J, F Rojo, G Sánchez. 2009. Los Palancares y Agregados: 111 años de gestión forestal sostenible. Comunidad de Castilla - La Mancha. 56 p. (Serie Forestal No 7).

Generalitat Valenciana. 1999. Los montes valencianos en cifras. Extracto del segundo inventario forestal de la Comunidad Valenciana. Edición Conselleria de Agricultura y Medio Ambiente. 51 p.

Generalitat Valenciana. 2004. Plan General de Ordenación Forestal de la Comunidad Valenciana. Valencia, España. Conselleria de Territori i Habitatge. 465 p.

Generalitat Valenciana. 2011. Plan de Acción Territorial Forestal de la Comunidad Valenciana. Valencia, España. Generalitat Valenciana. Consultado 15 sep. 2010. Disponible en

http://www.cma.gva.es/web/indice.aspx?nodo736\&idioma=C.

Godoy MM, G Defosséa, M Thren. 2007. Especies forestales promisorias para la diversificación de forestaciones en la Patagonia Argentina. Bosque 28(1): 25-32.

Gómez M. 2008. Sistema de generación eléctrica con pila de combustible de óxido sólido alimentado con residuos forestales y su optimización. Tesis doctoral. Madrid, España. Escuela Técnica Superior de Ingeniería Industrial, Univer- sidad Nacional de Educación a Distancia. 336 p.

González J, P Ibarz. 1998. Monte bajo irregular de encina: Caracterización silvícola. Sistemas y Recursos Forestales 7(1-2): 95-108.

Hapla F, J Saborowski. 1984. Überlegungen zur Wahl des Stichprobenumfangs bei Untersuchungen der physikalischen und technologischen Holzeigenschaften. Forstarchiv 55(4): $135-138$

IDAE (Instituto para la Diversificación y Ahorro de Energía, ES). 2007. Manuales de energías renovables: Energía de la biomasa. IDAE. Consultado 15 feb. 2011. Disponible en http:// www.idae.es/index.php/mod.documentos

IFN3 (Tercer inventario Nacional Forestal, ES). 2008. "Tercer inventario Nacional Forestal". 1997-2007. Ministerio de Medio Ambiente. Consultado 10 de abr. de 2011. Disponible en http://www.magrama.gob.es/es/biodiversidad/temas/ inventarios-nacionales/

IMIDA (Instituto Murciano de Investigación y Desarrollo Agrario y Alimentario, ES). 2012. Bioenergy production in rural areas. Consejería de Agricultura y Agua de la Región de Murcia. Consultado 23 de mayo de 2012. Disponible enhttp://www.imida.es/docs/Varios/Bioenergy_08092011/ Produccion_Bioenergia_zonas_rurales.pdf

Institute Pinchot. 2010. A Guide to Forest Biomass Harvesting and Retention in Maryland. Maryland, USA. Institute Pinchot . 25 p.

Márquez-Montesino F, T Cordero, J Rodríguez-Mirasol, J Rodríguez-Jiménez. 2001. Estudio del potencial energético de biomasa de Pinus caribaea Morelet var. caribaea (Pc) y Pinus tropicalis Morelet (Pt), Eucalyptus saligna Smith (Es), Eucalyptus citriodora Hook (Ec) y Eucalyptus pellita F. Muell (Ep): de la Provincia de Pinar del Rio. Chapingo. Ciencias Forestales y del Ambiente 7(1): 83-89 p.

Merino A, MA Balboa, R Rodríguez-Soalleiro, JG Álvarez-González. 2005. Nutrient exports under different harvesting regimes in fast-growing forest plantations in southern Europe. Forest Ecology and Management 207: 325-339.

MINETUR (Ministerio de Industria, Turismo y Comercio). 2010. Plan de Acción Nacional de Energías Renovables de España (National Action Plan For Renewable Energy In Spain). Madrid, España. Ministerio de Industria, Turismo y Comercio. 173 p.

Mouissa H, RA Fournier. 2013. Mapping stand volumes of Pinus halepensis Mill in a semi-arid region using satellite imagery of the Sénalba Chergui forest in north-central Algeria. Journal of Arid Environments 92: 63-75.

Mukhortova L. 2012. Carbon budget recovery and role of coarse woody debris in post-logging forest ecosystems of Southern Siberia. Bosque 33(3): 261-265.

Novo A. 2008. Factores que influyen en el aprovechamiento. Planificación del apeo manual. Aprovechamientos Forestales. In Escuela Universitaria de Ingeniería Técnica Forestal, Universidad de Vigo ed. Planificación del Aprovechamiento Maderero. 19-38 p..

Núñez-Regueira L, JÁ Rodríguez Añón, J Proupín Castiñeiras. 1996. Calorific values and flammability of forest species in Galicia. Coastal and hillside zones. Bioresource Technology 57(3): 283-289

Ortego J, R Bonal, A Muñoz. 2010. Genetic Consequences of Habitat Fragmentation in Long-Lived Tree Species: The Case of the Mediterranean Holm Oak (Quercus ilex L.) 
Journal of Heredity 101(6): 717-726.

Pérez P, Esteban L. 2004. Cuantificación de los recursos de biomasa forestal en la provincia de Soria y evaluación de alternativas para su aprovechamiento energético. Ciencia Técnica 75: 17-25.

Perry D. 1985. The competition process in forest stand. In Cannel MGR. JE Jackson eds. Attributes of trees as crop plants. Penicuick, United Kingdom. Institute of Terrestrial Ecology. p. 481-506.

PNW (Pacific Northwest, US). 2010. Reducing Fire Risk on Your Forest Property. 41 p. (Bulletin $n^{\circ} 618$ )

Prodan M, R Peters, F Cox, P Real. 1997. Mensura forestal. Serie de investigación y evaluación en desarrollo sostenible. San José, Costa Rica. IICA/GTZ. 561 p.

Pulido FJ, M Díaz, SJ Hidalgo de Trucios. 2001. Size structure and regeneration of Spanish holm oak Quercus ilex forests and dehesas: effects of agroforestry use on their long-term sustainability. Forest Ecology and Management 146: 1-13.

Rivas-Martínez S, A Penas Merino. 2003. Atlas y manual de los hábitats de España. Ministerio de Medio Ambiente y Medio Rural y Marino. Consultado 11 dic. 2010. Disponible en http://www.magrama.gob.es/es/biodiversidad/servicios

Rodà F, J Vayreda, M Ninyerola. 2009. Encinares de Quercus ilex y Quercus rotundifolia. Ministerio de Medio Ambiente y Medio Rural y Marino. Consultado 1 jun. 2011. Disponible en http://www.jolube.es/Habitat_Espana/documentos/9340.pdf.

Sachs L. 1984. Angewandte Statistik (practical statistics). Berlin, Germany. Springer Verlag. 552 p.

Serrada-Hierro R, A San Miguel. 2008. Selvicultura en dehesas. Consultado 21 oct. 2011. Disponible en http://www2. montes.upm.es/Dptos/DptoSilvopascicultura/SanMiguel/ publicaciones/2006-2010/2008_Selvicultura\%20de\%20dehesas_Compendio selvicultura_rev.pdf

Serrada-Hierro R, J A Bravo Fernández, S Roig Gómez. 2004. Brotación en encinas (Quercus ilex subsp. ballota) con edades elevadas. Experiencias en el monte de Riofrío (Segovia). Investigación Agraria. Sistemas y Recursos Forestales 13(4): 127-141.

Skilling D. 1990. Pinus sylvestris L. Scotch pine. In Burns RM, BH Honkala eds. Silvics of North America. Washington D.C, U.S.A. Department of Agriculture, Forest Service. p. 489-496.

Torres-Álvarez O, F Peña-Cortés. 2011. Zonificación del potencial energético de la biomasa residual forestal en la cuenca del lago Ranco, Chile. Antecedentes para la planificación energética regional. Bosque 32(1): 77-84.

Recibido: 10.10 .13

Aceptado: 24.01.14 